\title{
When Timing the Mind One Should Also Mind the Timing: Biases in the Measurement of Voluntary Actions
}

\author{
Steve Joordens, * Marc van Duijn, $\dagger$ and Thomas M. Spalek* \\ *University of Toronto at Scarborough, Scarborough, Ontario, Canada; and \\ †Leiden University, Leiden, The Netherlands
}

\begin{abstract}
Trevena and Miller (2002, this issue) provide further evidence that readiness potentials occur in the brain prior to the time that participants claim to have initiated a voluntary movement, a contention originally forwarded by Libet, Gleason, Wright, and Pearl (1983). In their examination of this issue, though, aspects of their data lead them to question whether their measurement of the initiation of a voluntary movement was accurate. The current article addresses this concern by providing a direct analysis of biases in this task. This was done by asking participants to make subjective timing decisions regarding a stimulus that could be measured objectively. Our findings suggest that their timing task was indeed biased such that participants' tend to report events as happening approximately $70 \mathrm{~ms}$ later than they actually happened. Implications for the original Libet et al. claims are discussed. (๑) 2002 Elsevier Science (USA)
\end{abstract}

As described throughout this special issue, the cortical stimulation and measurement studies of Libet and colleagues lead them to make many claims that seem to violate our experiential notions of consciousness. As one example, Libet $(1981 ; 1991)$ argues that conscious perception of our world does not actually occur "online" but rather lags up to $500 \mathrm{~ms}$ behind actual events. We do not sense this lag because subjective timing of the sensory experience is referred back to the moment of the cortical time-marker (primary evoked potential), which occurs in the somatosensory cortex 10-30 ms after peripheral stimulation (Dennett, 1991; Libet, 2000; Libet, Wright, Feinstein, \& Pearl, 1979). If this is true, then it suggests that conscious cognition cannot play an integral role in any fast reactions to the world, implying that conscious processes may be limited to control and selection of unconsciously initiated actions (Libet, 1985; Velmans, 2000).

The present article focuses on a related claim made by Libet and colleagues, the claim that when a person is asked to voluntarily initiate a behavior, indications of cortical movement preparation can be seen prior to the time the participant claims to subjectively initiate the movement (Libet, 1985). The implication of this claim is that while conscious processes may set up the conditions that would support a "voluntary" movement, the movement itself is actually initiated by unconscious processes and then information about the upcoming movement is forwarded to awareness prior to the movement occurring. Given that our awareness of the movement precedes

Address correspondence and reprint requests to Steve Joordens, Division of Life Sciences, University of Toronto at Scarborough, 1265 Military Trail, Scarborough, Ontario, Canada M1C 1A4. E-mail: joordens@psych.utoronto.ca. 
the actual movement, we feel that we consciously caused the act to occur (Wegner \& Wheatley, 1999).

As several of the articles in this issue have highlighted, the original data and procedures upon which Libet's claims are based are not as clean as one might like and, in many cases, alternative interpretations are possible if not preferable (Churchland, 1981a; 1981b; Dennett \& Kinsbourne, 1992; Glynn, 1990; Gomes, 1998, 2002; van de Grind \& Lokhorst, 2001; Pockett, 2002). However, while not agreeing with the original procedures upon which Libet et al.'s (1983) voluntary movement claims were based, Trevena and Miller (2002) did successfully replicate the critical finding upon which Libet et al.'s claims are based as had Keller and Heckhausen (1990). From our perspective, then, it is the claims concerning voluntary movements that are of primary interest given that they have now been independently replicated.

The purpose of the present study is to examine one issue that was discussed but not directly addressed in the Trevena and Miller (2002) study; specifically, the issue of potential report biases with respect to the clock-watching procedure used to measure the voluntary initiation of behavior. This issue is highly relevant because the critical finding from the Trevena and Miller study is the finding of readiness potentials occurring in the brain prior to the time when the participant claimed to initiate the behavior. If any biases were present that influenced participants to falsely report their voluntary initiation as occurring later than it actually had, then this critical finding could be due to these biases rather than to late conscious involvement. Thus, in order to properly interpret the finding of readiness potentials occurring prior to the initiation of a voluntary act, an analysis of potential biases in the reporting of the initiation time seems prudent.

\section{Brief Background}

In the original study of the initiation of voluntary movements conducted by Libet et al. (1983) participants were instructed to watch a round clockface that included a clockwise moving dot. At some point they were to initiate a voluntary movement and to note the location of the dot on the clock at that point in time. When brain recordings were then synchronized with the time the dot occurred in the reported location, readiness potentials were observed to occur prior to that time. This is an interesting finding indeed if the participant's report of the dot location associated with voluntary initiation is accurate. Libet realized the importance of verifying the accuracy of report using the clock stimulus and attempted to provide such verification by asking participants to similarly note the occurrence of a tactile stimulus whose onset could be objectively specified. Based on this experiment Libet claimed that the clock-watching task did provide accurate time estimates.

Trevena and Miller (2002) did not take issue with the clock-watching procedure but rather took issue with the use of a general readiness potential as an indicator of cortical movement preparation. Specifically, they argued that the readiness potential is sensitive to many cognitive processes other than simple movement preparation and, therefore, that the finding of readiness potentials does not necessarily imply that movement preparation was the cause. Instead they argued for the use of lateralized readiness potentials as a better indicator of cortical movement preparation, and they 
did a clock-watching experiment of their own using both readiness potentials and lateralized readiness potentials. Replicating the critical finding of Libet et al. (1983), they found that readiness potentials do occur prior to the reported initiation times; however, lateralized readiness potentials occurred after the initiation times. Based on their claims that it is the lateralized readiness potential that truly reflects motor preparation, they interpreted their findings as consistent with the notion that the movements were, in fact, voluntarily controlled.

While Trevena and Miller's (2002) interpretation suggests that we do have voluntary control of our initiations of movement, it does still leave the mystery of why readiness potentials are occurring prior to the time when participants claimed to have initiated their behavior. In considering this, one aspect of their data that they noted was that some of the reported initiation times actually occurred after the movement had occurred. This finding seems very bizarre unless there was some form of bias occurring for the reports of initiation times, a bias that influenced participants to report their initiation times as occurring later than they actually had. If such a bias were operating, it might also explain why readiness potentials can occur prior to the reported initiation times. That is, it may be the case that readiness potentials are actually coinciding with the voluntary initiation of behavior but then some bias influences the participants to report the initiation as happening later than it had, making the readiness potential appear to occur prior to the initiation.

\section{Potential Biases in the Clock-Watching Task}

Before going further it seems prudent to describe Trevena and Miller's (2002) clock-watching task in more detail. At the beginning of each trial either the letter L or the letter $\mathrm{R}$ was presented briefly, followed by a blank screen, followed by a tall, thin rectangular clock with the numbers 1 through 12 evenly spaced along its perimeter. A dot moved at a fairly quick rate in a clockwise direction around the perimeter and, at some point of the participant's choosing, they were to initiate a keypress noting the position of the dot at the time they decided to initiate the movement. If the initial letter was an L they were to press a key with their left hand, if it was an $\mathrm{R}$ they were to press a key with their right hand. The right versus left hand manipulation was required for the meaningful analysis of lateralized readiness potentials but is not relevant to our points.

Once the key was pressed the dot continued to move for a short period (500-800 $\mathrm{ms}$ ) and was then removed and a referent dot was presented. At this point the participant was to indicate whether he or she had initiated their movement at a point either before the location of the referent or after the location of the referent. This temporal order judgment was then used to locate the point at which the participant believed they initiated the behavior, and it was this point that was then compared to the readiness potential and the lateralized readiness potential.

One thing we wish to emphasize about this task is that it is not so much a perceptual task as it is a memory task, and a challenging memory task at that. As mentioned, the dot is moving quickly, and it continues to move after the participant decides to initiate the behavior, even continuing to move for a while after the keypress is made. Thus, the participant is first asked to remember one specific position of a moving 
target while simultaneously performing a choice response task and is then asked to compare this memory with a referent to make the binary before/after decision.

Conceptualized as a two-alternative memory task, it is easier to consider potential sources of biases in the clock-watching procedure. Specifically, the clock procedure is similar to procedures that elicit a memory bias termed "the representational momentum effect" (e.g., Hubbard \& Barucha, 1988). In addition, there may also be a decision bias operating in this task given the relative frequency of correct "before" versus "after" responses. These two possibilities are now discussed in more detail, making explicit the manner in which they could add a constant to the reported decision times.

Turning first to the potential for a memory bias, a number of experiments have now documented the representational momentum effect (Finke \& Freyd, 1985; Finke, Freyd, \& Shyi, 1986; Freyd \& Finke, 1984, 1985; Hubbard, 1990, 1995; Hubbard \& Barucha, 1988). This effect occurs when participants are asked to remember the location (or orientation) of an item whose location (or orientation) is changing in a regular manner. For example, Hubbard and Barucha (1988) had a small circular target smoothly moving either horizontally or vertically across the computer screen. At some point the target would disappear from the screen, and the participant was asked to move a mouse cursor to the vanishing point and press the mouse button. The results of this experiment showed that participants overestimated the final position of the target consistent with the implied motion of the target. This situation is quite similar to that of the clock-watching task in that participants are asked to remember the location of a dot moving in a regular clockwise manner. If some form of representational momentum is operating in the clock-watching task, then one would expect participants' memory to be biased such that they would actually remember the dot being further along its trajectory than it really was. Such a bias would give the impression that the volitional act was initiated later than it actually was.

Similarly, if one also considers the decision dynamics of the task there is another potential source of bias. In the Trevena and Miller (2002) study, the position of the referent dots was determined using an interleaved staircase method. The details of this method are presented in the original article, and there is no need for us to reiterate them here. However, one aspect of their locations is important. Based on our understanding of their procedure, if the participant actually had a veridical memory of the location of the dot at the time of their volitional initiation of a response, this position would be prior to the location of the referent dot on the minority of trials. Thus, if participants were to choose their "before" versus "after" responses based on sensitivity alone, they would be making relatively few "before" responses. However, when two response alternatives are present, participants are hesitant to use only one of them on the majority of trials, especially in challenging memory contexts. It is therefore possible that participants may opt to use the "before" response more in situations where they were unsure of the correct response which could inflate the errant proportion of "before" responses. That is, participants may be influenced to say "before" in situations where their veridical memory was actually after the referent. The result of this would again be that the positioning of the volitional act would be further downstream than it really was, leaving room for readiness potentials to apparently occur prior to the volitional act. 
Thus, biases in either memory or in the decision process may have lead participants to report their point of initiation as being later than it actually was. If this is the case, then readiness potentials that occurred earlier than the reports of initiation may not have occurred earlier than the actual initiation. Clearly a close empirical look at biases in the clock-watching task is warranted.

\section{EXPERIMENT}

The purpose of our experiment was to assess whether there are biases in the clockwatching task as used by Trevena and Miller (2002). To this end we were careful to replicate all details of the task that we viewed as relevant given our analysis above. The primary difference though was that rather than having participants indicate when they initiated an action, we asked them to indicate when the clock perimeter changed color. The notion is that by using an objectively measurable stimulus we can directly assess the ability of the participants to fix that event in time and directly measure any biases that may be inherent in that judgment.

\section{Method}

Participants. The participants were the second author of this article and five undergraduate students from the University of Toronto at Scarborough. The undergraduates were given either course credit or $\$ 10$ Canadian in exchange for their participation. All participants had normal or correct-to-normal vision.

Procedure. Our procedure was modeled directly after that used by Trevena and Miller (2002) with only slight variations. Specifically, there were 12 blocks of 20 trials each. Each trial consisted of the following sequence of events: (1) the letter L or R was presented at the vertical and horizontal center of the screen for $1 \mathrm{~s}$, (2) a blank screen was presented for a duration randomly chosen from a uniform distribution ranging from 1 to $2 \mathrm{~s}$, (3) a blue clock measuring $15 \mathrm{~mm}$ wide and $110 \mathrm{~mm}$ high was presented with the numbers 1 through 12 arranged around its perimeter at equal intervals and with associated tick marks, (4) a white dot appeared at a randomly chosen location on the clock's perimeter and began moving in a clockwise direction at a rate of 8 revolutions per $3.25 \mathrm{~s}$, (5) after a randomly determined time sampled from a uniform distribution ranging from 2 to $8 \mathrm{~s}$ the clock perimeter changed from blue to yellow, (6) the dot continued to move while the program waited for the participant to hit either the " $z$ " (L response) or " "/" (R response) key to indicate they had noted the color change, (7) the dot continued to move for a duration randomly sampled from a uniform distribution ranging from 500 to $800 \mathrm{~ms}$, (8) a referent dot was presented at a location as specified below, (9) the referent dot remained visible until participants pressed either " $x$ " (to indicate a "before" response) or "." (to indicate an 'after" response), and (10) a 500-ms blank screen followed by automatic initiation of the next trial.

Participants were instructed to watch the clock and note where the dot was when the perimeter of the clock changed from blue to yellow. At that point they were to press the key indicated at the beginning of the trial (i.e., L or R). They were told that shortly thereafter the dot would stop moving and would then appear at another location on the clock. They were to use this dot as a referent and to then indicate 
whether their remembered location was before or after the referent location by pressing the appropriate key. Note that this procedure is identical to that used by Trevena and Miller (2002) except that in place of participants noting when they decided to initiate a response, participants in the current experiment were asked to note when the clock turned yellow.

We also altered the manner in which the referent dot location was chosen. Trevena and Miller (2002) determined the referent location using an interleaved staircase method that was somewhat complicated and that did not insure that the referent dot would often occur at locations close to the color change location. Given that these were the locations we were primarily interested in, and given we saw no reason why the staircase method would introduce biases of its own, we used a less complicated method for determining the referent location. Specifically, the location of the referent dot was randomly determined by sampling from a uniform distribution that covered a range from $67 \mathrm{~ms}$ prior to change in color (i.e., four 16.7-ms steps before the step where the color changed) to $67 \mathrm{~ms}$ after the L/R response. By using this range we were able to examine the probability of "before" responses around both the color change location and the keypress location. In all other respects we made every attempt to match our procedural details to those described by Trevena and Miller (2002).

\section{Results and Discussion}

We present our data via two illustrations. Following the lead of Trevena and Miller (2002, Fig. 4) the first examines the probability of participants' providing a "before" response as a function of the referent's distance from the point where they made their L/R response. Recall that a "before" response corresponds to trials on which the participant believed that the color changed had occurred before the point indicated by the referent dot. Given that the color change always occurred before the L/R response, one would expect the probability of a "before" response to grow as one moves closer in time to the keypress. As illustrated in Fig. 1, this is exactly what was observed.

Although this figure is not critical to the points we argue, it does illustrate two things. First, the growth curve presented in Fig. 1 is highly similar to that presented in Figure 4 of Trevena and Miller, suggesting that our participants were performing the task in a manner similar to theirs. Second, given that the probabilities do grow as expected this suggests that the participants understood the instructions and were making their "before" versus "after" decisions appropriately.

For current purposes the more relevant data are presented in Fig. 2. Figure 2 shows the probability of making a "before" response as a function of how far the referent dot was from the actual point where the clock turned yellow. In such a situation, the point where participants are saying "before" with a probability of .5 is the point of "subjective equality." That is, this is the point where a participant is as likely to say "before" as "after" and, as such, it is assumed to reflect the location where the participant remembered the event as occurring.

As illustrated in Fig. 2, the point of subjective equality does not occur at the actual color change time but rather occurs approximately four steps beyond that point. In fact, by fitting a line to Fig. 2 and using it to find the 0.5 point we can conclude that, 


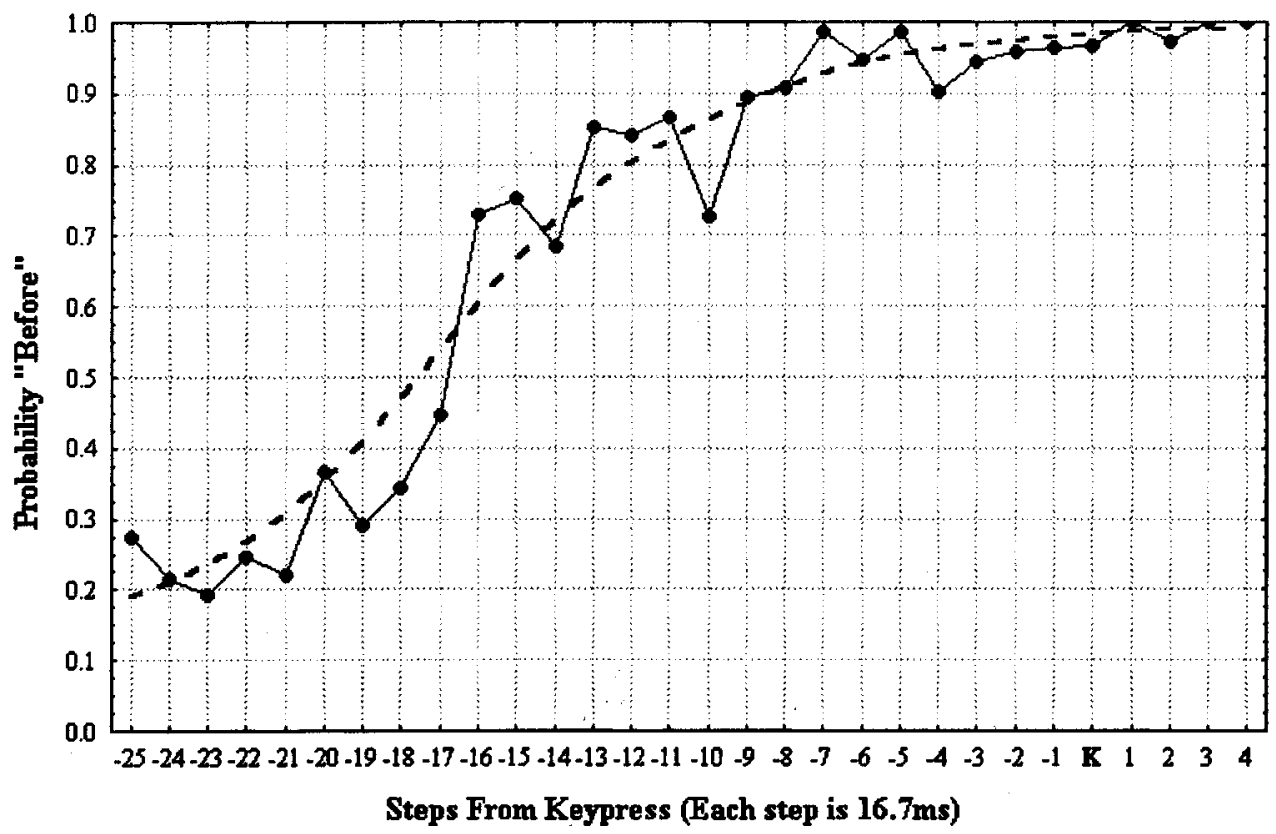

FIG. 1. The proportion of "before" responses as a function of the position of the referent dot relative to the keypress (bold K). Each step represents one movement of the dot, and one movement of the dot occurred each $16.7 \mathrm{~ms}$. The overlaid dotted line represents the least-squared fit to the data.

on average, participants in our study remember the color change occurring 65-70 ms later than it actually had. Thus, reports obtained using the clock-watching procedure are biased, and the bias is the type that moves the report further ahead in time.

\section{GENERAL DISCUSSION}

Trevena and Miller (2002) provide a replication and extension of the findings originally reported by Libet et al. (1983). Specifically, they replicated the finding that readiness potentials do occur prior to the participants' reported time of initiating a voluntary movement. However, they also showed that lateralized readiness potentials tend to occur after the reported initiation time. Given their claims that lateralized readiness potentials are the best indicator of movement preparation, they interpreted their findings as consistent with the notion that motor acts can be voluntarily controlled.

If we accept their interpretation at face value we are still left with the question of what the readiness potential is then reflecting. If this potential does not reflect motor preparation, what then what does it reflect? Trevena and Miller (2002) entertain some answers to this question, one of which is relevant to our work. That is, they suggested that while readiness potentials may be occurring prior to the "reported" initiation time, it is possible that there is bias in this report that pushed it further ahead in time than when the actual event occurred.

In the current article we provided evidence that using the clock-watching task, 


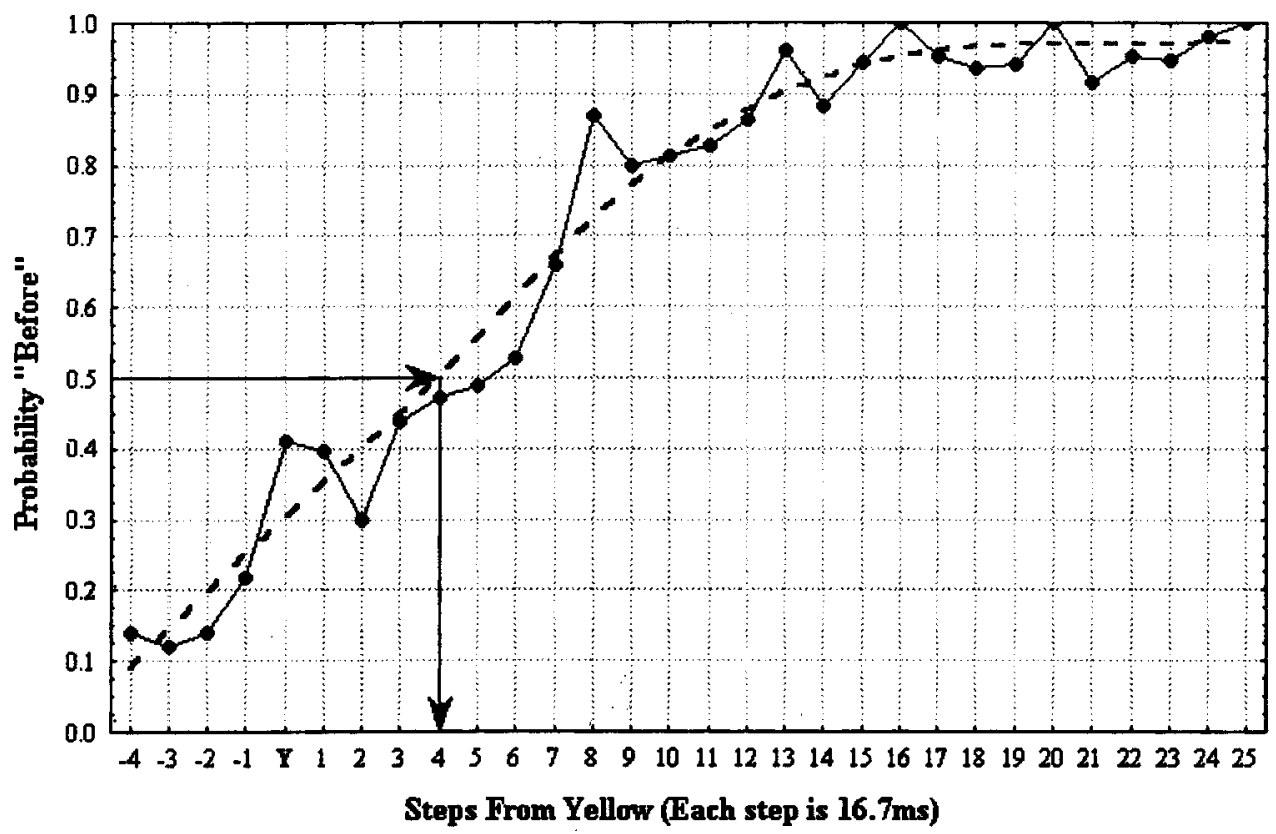

FIG. 2. The proportion of "before" responses as a function of the position of the referent dot relative to the point where the clock changed from blue to yellow (bold Y). Each step represents one movement of the dot, and one movement of the dot occurred each $16.7 \mathrm{~ms}$. The overlaid dotted line represents the least-squared fit to the data.

reports of the occurrence of objectively measurable stimulus were biased such that participants remembered it as occurring later than it actually had. As described in the introduction, the presence of this bias is actually not surprising given the details of the procedure, and it could be due to a memory bias (i.e., a representational momentum effect), a decision bias (i.e., participants attempting to match what they assume to be the a priori probability of before versus after trials), or perhaps a mixture of the two.

The critical implication of this is that it provides a possible alternative account of the Libet et al. (1983) data. That is, if participants actually initiated the behavior earlier than their report would indicate, then it is possible that the readiness potential that was observed is actually associated with the true initiation of the response. This initiation was then remembered as occurring later than it actually had, making it appear as though the readiness potential occurred prior to the voluntary initiation of the movement.

A second possibility is that the bias documented here is only part of the story. That is, we showed evidence of a bias on the order of $70 \mathrm{~ms}$. However, in some cases (e.g., Trevena \& Miller, 2002, Experiment 2) the readiness potential actually occurred up to $2 \mathrm{~s}$ prior to the movement! Moreover, numerous studies have also shown that the readiness potential is sensitive to many factors other than movement preparation. It would be interesting to know whether the readiness potential would 
occur more than $70 \mathrm{~ms}$ prior to the voluntary initiation of the response in a context where these other factors were controlled.

\section{REFERENCES}

Churchland, P. S. (1981a). On the alleged backwards referral of experiences and its relevance to the mind-body problem. Philosophy of Science, 48, 165-181.

Churchland, P. S. (1981b). The timing of sensations: Reply to Libet. Philosophy of Science, 48, 492497.

Dennett, D. C. (1991). Consciousness explained. London/New York: Penguin Books.

Dennett, D.C., \& Kinsbourne, M. (1992). Time and the observer: The where and when of consciousness in the brain. Behavioral and Brain Sciences, 15, 183-247.

Finke, R. A., \& Freyd, J. J. (1985). Transformations of visual memory induced by implied motions of pattern elements. Journal of Experimental Psychology: Learning, Memory, and Cognition, 11, 780794.

Finke, R. A., Freyd, J. J., \& Shyi, G. C. W. (1986). Implied velocity and acceleration induce transformations of visual memory. Journal of Experimental Psychology: General, 115, 175-188.

Freyd, J. J., \& Finke, R. A. (1984). Representational Momentum. Journal of Experimental Psychology: Learning, Memory, and Cognition, 10, 126-132.

Freyd, J. J., \& Finke, R. A. (1985). A velocity effect of representational momentum. Bulletin of the Psychonomic Society, 23, 443-446.

Glynn, I.M. (1990). Consciousness and time. Nature, 348, 477-479.

Gomes, G. (1998). The timing of conscious experience: A critical review and reinterpretation of Libet's research. Consciousness and Cognition, 7, 559-595.

Gomes, G. (2002). Problems in the timing of conscious experience. Consciousness and Cognition, 11, 191-197.

van de Grind, W. A. \& Lokhorst, G. J. C. (2001). Hersenen en bewustzijn: van pneuma tot grijze massa. In F. Wijnen \& F. Verstraten (Eds.), Het brein te kijk: verkenning van de cognitieve neurowetenschappen, pp. 217-246. Lisse: Swets en Zeitlinger.

Hubbard, T. L. (1990). Cognitive representation of linear motion: Possible direction and gravity effects in judged displacement. Memory \& Cognition, 18, 299-309

Hubbard, T. L. (1995). Environmental invariants in the representation of motion: Implied dynamics and representational momentum, gravity, friction, and centripetal force. Psychonomic Bulletin \& Review, 2, 322-338.

Hubbard, T. L., \& Barucha, J. J. (1988). Judged displacement in apparent vertical and horizontal motion. Perception \& Psychophysics, 44, 211-221.

Keller, I., \& Heckhausen, H. (1990). Readiness potentials preceding spontaneous motor acts: Voluntary versus involuntary control. Electroencephalography and Clinical Neurophysiology, 76, 351-361.

Libet, B. (1981). The experimental evidence for subjective referral of sensory experience backwards in time: Reply to P. S. Churchland. Philosophy of Science, 48, 182-197.

Libet, B. (1985). Unconscious cerebral initiative and the role of conscious will in voluntary action. The Behavioral and Brain Sciences, 8, 529-566.

Libet, B. (1991). Conscious vs. neural time. Nature, 352, 27-28.

Libet, B. (2000). Time factors in conscious processes: A reply to Gilberto Gomes. Consciousness and Cognition, 9, 1-12.

Libet, B., Gleason C. A., Wright, E W., \& Pearl, D. K. (1983). Time of conscious intention to act in relation to onset of cerebral activity (Readiness-potential): The unconscious initiation of a freely voluntary act. Brain, 106, 623-642.

Libet, B., Wright, E. W., Jr., Feinstein, B., \& Pearl, D. K. (1979). Subjective referral of the timing for 
a conscious sensory experience: A functional role for the somatosensory specific projection system in man. Brain, 103, 193-224.

Pockett, S. (2002). On subjective back-referral and how long it takes to become conscious of a stimulus: A reinterpretation of Libet's data. Consciousness and Cognition, 11, 144-161.

Trevena, J. A., \& Miller, J. (2002). Cortical movement preparation before and after a conscious decision to move. Consciousness and Cognition, 11, 162-190.

Velmans, M. (2000). Understanding consciousness, London: Routledge/Psychology Press/Taylor \& Francis.

Wegner, D. M., \& Wheatley, T. P. (1999). Apparent mental causation: Sources of the experience of will. American Psychologist, 54, 480-492. 